\title{
CAMBIOS EN EL ESTILO DE VIDA Y EN LAS EMOCIONES DURANTE EL CONFINAMIENTO POR COVID-19
}

\author{
Luisa Marilia Cantisano \\ Pontificia Universidad Católica Madre y Maestra (PUCMM) República Dominicana. \\ Universidad Europea de Madrid. \\ Noelia Belando Pedreño \\ Universidad Europea de Madrid \\ Ángel Ballester Sánchez \\ Functional Feel training, Madrid \\ Ascensión Blanco Fernández \\ Universidad Europea de Madrid \\ Rocío González-Soltero $\mathbf{b}$ * \\ Universidad Europea de Madrid \\ mariadelrocio.gonzalez@universidadeuropea.es
}

Recepción Artículo: 16 mayo 2021 Admisión Evaluación: 17 mayo 2021 Informe Evaluador 1: 26 mayo 2021 Informe Evaluador 2: 28 mayo 2021 Aprobación Publicación: 02 junio 2021

\section{RESUMEN}

En marzo de 2020, la pandemia de la COVID-19 hizo que, a lo largo del mundo, muchos países adoptaran medidas basadas en confinamientos domiciliarios. El objetivo era detener la propagación, así como la crisis hospitalaria y las muertes derivadas de la infección por SARS-CoV-2. Las personas tuvieron que reajustar sus hábitos de vida para adaptarse a la nueva situación. El objetivo principal de esta investigación fue evaluar la percepción poblacional sobre los cambios en el estilo de vida experimentados durante su confinamiento domiciliario en torno a cuatro parámetros: (a) su alimentación, (b) la frecuencia/intensidad de ejercicio físico, (c) el estado emocional y (d) el uso de medios digitales de salud digital (eSalud). Con este objetivo, el 31 de marzo y el 1 de abril, se envió el enlace de una encuesta diseñada en Formularios de Google a través de WhatsApp. Se reclutó una muestra de 320 participantes, con edades comprendidas entre los 15 y los 78 años (=32.97; $s=11.51$ ), residentes de distintos países americanos y europeos, principalmente de República Dominicana (73.8\%) y España (17.2\%). Los resultados evidenciaron, respecto a la alimentación, que el 27,2\% sentía que su alimentación había empeorado (37.8\% que era igual, mientras que 35,0\% que incluso había mejorado); en cuanto al ejercicio, $52.5 \%$ manifestaba que había disminuido su frecuencia/intensidad (23.1\% que era igual, mientras que $24.4 \%$ que había aumentado); a nivel emocional, $34.4 \%$ señaló que había empeorado (51.6\% que era igual, mientras que 
14.0\% que era más positivo). Se observó una asociación estadísticamente significativa entre los cambios en el uso de herramientas de eSalud en la alimentación $\left(c^{2}[4, n=320]=11.75, p=.019\right)$ y el ejercicio físico $\left(c^{2}[4, n\right.$ $=320]=85.35, p=.000$ ). No se observó asociación estadísticamente significativa entre los cambios emocionales y en el uso de herramientas de eSalud.

Palabras clave: alimentación; ejercicio físico; emociones; COVID-19; eSalud

\section{ABSTRACT}

Changes in lifestyle and emotions during the COVID-19 lockdown. In March 2020, the COVID-19 pandemic caused many countries around the world to adopt measures based on home containment. The aim was to stop the spread, as well as the hospital crisis and deaths resulting from SARS-CoV-2 infection. People had to readjust their living habits to adapt to the new situation. The main objective of this research was to assess the population perception of lifestyle changes experienced during their home confinement around four parameters: (a) their diet, (b) frequency and intensity of physical exercise, (c) emotional state and (d) use of digital health digital media (eHealth). On March 31 and April 1, 2020, the link to a survey designed specifically for this purpose on Google Forms was sent via WhatsApp. A sample of 320 subjects was recruited, aged between 15 and 78 years $(=32.97 ; s=11.51)$, residents of different American and European countries, mainly from the Dominican Republic (73.8\%) and Spain (17.2\%). The results evidenced, with respect to diet, that $27.2 \%$ felt that their diet had worsened (37.8\% that it was the same, while 35.0\% that it had even improved); with respect to exercise, $52.5 \%$ stated that it had decreased in frequency/intensity (23.1\% that it was the same, while $24.4 \%$ that it had increased); at the emotional level, 34.4\% stated that it had worsened (51.6\% that it was the same, while 14.0\% that it was more positive). A statistically significant association was observed between changes in the use of eHealth tools in eating $\left(c^{2}[4, n=320]=11,75, p=.019\right)$ and exercise $\left.c^{2}[4, n=320]=85,35, p=.000\right)$. No statistically significant association was observed between emotional changes and the use of eHealth tools.

Keywords: diet; physical exercise; emotions; COVID-19; eHealth

\section{INTRODUCCIÓN}

En diciembre de 2019, se reportó un brote de infección por SARS-CoV-2 en Wuhan, China. A partir de ahí, el virus se fue transmitiendo hasta llegar a la categoría de pandemia mundial, declarada en marzo de 2020. La Organización Mundial de la Salud (OMS, 2020) incentivó a los gobiernos y organismos sanitarios a tomar fuertes medidas para controlar la propagación de la COVID-19, las muertes y los colapsos hospitalarios que estaba generando. Estas medidas de distanciamiento social implicaron, en la mayoría de los países, confinamiento domiciliario y cierre de espacios públicos como centros educativos o gimnasios.

Con medidas semejantes, en epidemias pasadas se observaron efectos negativos a nivel psicológico: aumento de tasas de suicidio, ansiedad y deterioro del bienestar en general (Jeong et al., 2016), y muchos especialistas se han hecho eco de los efectos que, a largo plazo, podrían traer estos confinamientos.

Ahora bien, algo positivo de esta pandemia ha sido el interés creciente y significativo en aspectos relacionados con la salud, la alimentación o el ejercicio físico. Una revisión reciente sobre el impacto del confinamiento provocado por la COVID-19 sobre los hábitos alimentarios a nivel mundial (Bennet et al., 2021), concluye que este dio lugar a cambios tanto favorables (mayor preocupación por la alimentación), como desfavorables (menor consumo de alimentos frescos) en las prácticas alimentarias, y esto puede tener consecuencias a corto y largo plazo en la salud. Es de señalar que la mayoría de los estudios analizados por Bennet et al. incluyeron poblaciones europeas, por lo que resulta de interés saber el impacto en otras poblaciones. Aunque más de la mitad de los estudios incluían poblaciones europeas, hay pruebas que sugieren que es necesario actuar a nivel mundial para animar a la gente a volver a adoptar hábitos de vida saludables durante y después del confinamiento.

En relación al ejercicio físico, algunos estudios como el llevado a cabo en Bélgica por (Constandt et al., 2020) basado en una encuesta en línea, han examinado los niveles y patrones de ejercicio de los adultos durante el cierre de COVID-19. Estos análisis reflejan un resultado satisfactorio en cuanto a las frecuencias de ejercicio 
especialmente en la población que era más activa antes del confinamiento, pero también un incremento del comportamiento sedentario.

El uso de la salud digital (eSalud) podría ser una herramienta promotora de salud y cambios favorables en el estilo de vida (Marchant et al., 2021), muy adaptada a la situación de confinamiento. Según estos autores, la eSalud ofrece posibilidades para mantenerse activo, pero sus beneficios y los mecanismos psicológicos que resultan afectados están aún por demostrar, aunque actualmente existen herramientas de eSalud específicas que podrían adaptarse a cada persona y contexto. De hecho, existe evidencia epidemiológica sobre la importancia de integrar la atención a la salud mental y la eSalud en la planificación e implementación de políticas sanitarias, especialmente en colectivos sensibles como las embarazadas (Yang et al., 2021).

Por todo lo mencionado anteriormente, resulta esencial conocer el impacto que los confinamientos han tenido sobre la alimentación, el ejercicio físico y la salud mental de las personas, así como el papel que diferentes herramientas de eSalud podrían tener en favor de las mismas. Para ello, los objetivos de este estudio fueron, por un lado, evaluar los cambios percibidos por la población, durante el confinamiento por COVID-19, en torno a: calidad de su alimentación, frecuencia/intensidad de ejercicio físico y emociones, y, en segundo lugar, estudiar la asociación entre el uso de medios digitales (eSalud), y los cambios en el estado emocional, alimentación y ejercicio en el confinamiento.

\section{MATERIALES Y MÉTODOS}

\section{Comité de Ética}

El presente estudio ha sido aprobado por los Comité de Ética de la Investigación de la Universidad Europea de Madrid (España; código de aprobación CIPI/19/148), y el Comité de Bioética de la Facultad de Ciencias de la Salud de la Pontificia Universidad Católica Madre y Maestra (República Dominicana).

\section{Diseño de investigación}

El estudio que aquí se plantea tiene un enfoque de tipo descriptivo, predominantemente cuantitativo (Hernández Sampieri et al., 2014). El objetivo principal del mismo fue identificar, mediante encuesta en línea, cambios subjetivos en los patrones de alimentación, de ejercicio físico y estado emocional de los participantes, así como en el uso de herramientas de eSalud, durante la primera fase del confinamiento por COVID-19 (marzoabril 2020), en comparación con su situación antes de la pandemia.

\section{Tamaño muestral}

La población bajo estudio fue población hispanohablante que estuviese dispuesta a contestar un cuestionario en línea. El muestreo empleado fue no probabilístico por bola de nieve. Para reclutar la muestra, todos los investigadores enviaron el enlace del cuestionario a sus contactos de WhatsApp, tanto por medio de los chats grupales como individuales. Además, se solicitó a los receptores del mensaje que compartieran dicho enlace con otras personas. Con este procedimiento, se observó una muestra total de 320 participantes.

\section{Participantes}

El estudio estuvo formado por 320 participantes que respondieron al primer cuestionario, con edades comprendidas entre 15 y 78 años ( $=32.97 ; s=11.51)$. En la Tabla 1 se muestran los porcentajes para las variables sexo y país de residencia, siendo mayoritario el sexo femenino (73.1\%) y de procedencia República Dominicana $(73.8 \%)$. 
Tabla 1. Datos sociodemográficos de los participantes de la encuesta sobre emociones, alimentación, ejercicio físico y uso de eSalud durante la primera fase del confinamiento por COVID-19.

\begin{tabular}{lcc}
\hline \multicolumn{1}{r}{ Características } & $\boldsymbol{f}$ & $\mathbf{\%}$ \\
\hline Sexo & 234 & 73.1 \\
Femenino & 86 & 26.9 \\
Masculino & & \\
País de residencia & 236 & 7.8 \\
República Dominicana & 55 & 17.2 \\
España & 22 & 6.9 \\
Estados Unidos & 1 & 0.3 \\
Alemania & 1 & 0.3 \\
Canadá & 1 & 0.3 \\
Chile & 1 & 0.3 \\
Cuba & 1 & 0.3 \\
Francia & 1 & 0.3 \\
México & 1 & 0.3 \\
Panamá & &
\end{tabular}

Nota: $f=$ frecuencias.

\section{INSTRUMENTOS}

Para recoger información sobre los patrones de alimentación, ejercicio físico, estado emocional y uso de eSalud durante la primera fase del confinamiento por COVID-19, se empleó y creó el Cuestionario sobre cambio de hábitos y uso de mHealth COVID-19. Este fue un instrumento creado ad-hoc, a través de Formularios de Google, que incluye cuatro preguntas sociodemográficas, dos sobre los cambios percibidos por los participantes en su alimentación durante el confinamiento, una en torno a los cambios en el nivel de actividad física, dos acerca de las variaciones en el estado de ánimo/emociones, mientras que el resto estuvo orientado a evaluar el cambio en el uso de aplicaciones móviles y medios virtuales/digitales, así como los nombres específicos de lo usado en cada uno de los ámbitos bajo estudio. Se puede tener acceso al cuestionario a través del enlace: https://forms.gle/13EUvWPLVNWjTimj7

A todos los que completaron dicho cuestionario, se les remitió la Guía de Buenos Hábitos: Cuidando mi Salud durante el Confinamiento, documento de 20 páginas, dividido en tres secciones principales: 1) Recomendaciones para adaptar y mejorar la alimentación durante el confinamiento; 2) Recomendaciones para cuidar/mejorar el bienestar psicológico durante el confinamiento; 3) Recomendaciones para ejercitarse durante el confinamiento (https://drive.google.com/file/d/1z-QDeb0iT6Hb_0Zdq1mlsadzuh97fNG1/view?usp=sharing).

Tras cumplimentarlo, se les solicitó una retroalimentación por medio de otro cuestionario ad-hoc creado en Formularios de Google: Retroalimentaciones sobre "Guía de Buenos Hábitos: Cuidando mi Salud durante el Confinamiento" (https://forms.gle/13EUvWPLVNWjTimj7). Éste contiene 14 preguntas tipo Likert, de cinco opciones, para recopilar información acerca de la utilidad de cada una de las secciones de la guía (alimentación, ejercicio y regulación emocional). Además, incluye tres preguntas cerradas para evaluar la frecuencia de uso de la guía durante el confinamiento, así como tres preguntas abiertas para obtener sugerencias sobre cómo mejorar cada una de las secciones.

\section{PROCEDIMIENTO}

La difusión principal del Cuestionario sobre cambio de hábitos y uso de mHealth COVID-19 se hizo en los días 31 de marzo de 2020 y 1 de abril de 2020, con recordatorios para completarlo hasta el 13 de abril de 2020. Se ha de tener en cuenta que las medidas de cuarentena y confinamiento, en la mayoría de los países, comenzaron a principios o mediados de marzo de 2020. Desde la recepción de los resultados iniciales, se comenzó a elaborar 
la Guía de Buenos Hábitos: Cuidando mi Salud durante el Confinamiento. Esta tenía como objetivo contribuir a la mejora de los hábitos de los participantes y su estado emocional durante el confinamiento. Fue remitida el 22 de abril de 2020 a todas las personas que completaron el Cuestionario sobre cambio de hábitos y uso de mHealth COVID-19. El envío se realizó por correo electrónico colectivo con copia oculta a todos los participantes. El cuestionario de Retroalimentaciones sobre "Guía de Buenos Hábitos: Cuidando mi Salud durante el Confinamiento" fue enviado el 4 de mayo de 2020, a la misma lista de correos. En la Figura 1 se observa el diagrama de flujo de este procedimiento.

Figura 1. Diagrama de flujo de procedimiento, así como los instrumentos de medida creados para este estudio, así como el número de retroalimentaciones conseguidas en cada paso.

Elaboración de Formulario de Google 1 (Cuestionario sobre cambio de hábitos y uso de mHealth CoVID-19)



\section{Análisis estadístico}

Todos Ios análisis, descriptivos e inferenciales, se realizaron con el paquete SPSS v25.0. Para evaluar la asociación entre los cambios en la frecuencia de uso de herramientas de eSalud y la valoración subjetiva de los cambios en las otras variables del estudio (alimentación, ejercicio físico y emociones) se realizó una prueba de independencia de Chi-cuadrado $\left(c^{2}\right)$, así como una prueba de comparación de proporciones.

\section{RESULTADOS}

Descripción de las emociones, la alimentación y el ejercicio, así como de los cambios percibidos en esos aspectos, durante la fase inicial del confinamiento por covid-19.

En la Gráfico 1 se muestra la percepción que los encuestados tenían en torno a cómo habían cambiado su alimentación, hábitos de ejercicio físico y estado emocional durante las primeras semanas del confinamiento por COVID-19. El 37.8\% de los participantes encuestados sostuvo que su alimentación se había mantenido semejante al periodo previo al confinamiento, mientras que el menor porcentaje dijo que había empeorado (27.2\%). Respecto al ejercicio físico, la mayoría (52.5\%) indicó que la frecuencia y/o la intensidad de su ejercicio físico había disminuido durante este periodo. En la parte emocional, la mayoría planteó que no había notado cambios significativos en su estado de ánimo o emociones (51.6\%). No obstante, también es importante resaltar que solo un mínimo dijo que era más positivo antes del confinamiento (14.1\%). 
Gráfico 1. Comparación subjetiva de la calidad de los hábitos de vida y las emociones antes y durante las primeras semanas de confinamiento por COVID-19.

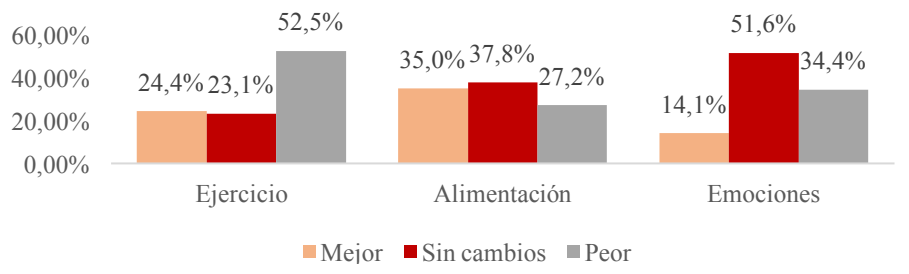

Posteriormente se preguntó a los participantes que identificaran los cambios positivos y negativos percibidos respecto a la alimentación durante el confinamiento. Esta parte del cuestionario fue rellenada por 199 participantes. Como se puede observar en la Tabla 2, en respuesta a la percepción de hábitos positivos durante el confinamiento, aquellos que percibieron mejoría en los hábitos de alimentación destacaron la mayor ingesta de comida casera (23.1\%), alimentos más saludables (22.6\%), y que tenían un mayor control y una organización más amplia de sus ingestas (17.6\%). Entre los aspectos nutricionales negativos a resaltar están: aumento del consumo de comida basura 0 de baja calidad nutricional (24.6\%), el aumento de la cantidad de alimentos (20.1\%), así como una desorganización en cuanto a las horas o las cantidades de ingestas que se hacían en un día (15\%).

Tabla 2. Cambios en los hábitos alimentarios durante el confinamiento por COVID-19.

\begin{tabular}{|c|c|c|}
\hline $\begin{array}{c}\text { Cambios alimentarios resaltados por los } \\
\text { participantes }(n=199)\end{array}$ & $f$ & $\%$ \\
\hline \multicolumn{3}{|l|}{ Aspectos positivos } \\
\hline Mayor ingesta de comida casera & 46 & 23.1 \\
\hline Mayor ingesta de comidas saludables & 45 & 22.6 \\
\hline Organización en horarios y/o número de ingestas & 35 & 17.6 \\
\hline Menor ingesta de comida de baja calidad nutricional & 23 & 11,6 \\
\hline Dieta más balanceada y variada & 19 & 9.5 \\
\hline Mayor ingesta de frutas y/o vegetales & 17 & 8.5 \\
\hline Reducción de porciones (antes se comía de más) & 10 & 5.0 \\
\hline Incremento necesario en cantidad de comida & 7 & 3.5 \\
\hline Menor ingesta de alcohol & 2 & 1.0 \\
\hline Mejor hidratación & 2 & 1.0 \\
\hline \multicolumn{3}{|l|}{ Aspectos negativos } \\
\hline $\begin{array}{l}\text { Mayor consumo de comida de baja calidad } \\
\text { nutricional }\end{array}$ & 49 & 24.6 \\
\hline Ingestas marcadamente aumentadas de alimentos & 40 & 20.1 \\
\hline Desorganización en horarios y/o número de ingestas & 30 & 15.0 \\
\hline Desbalance nutricional & 18 & 9.0 \\
\hline Menor ingesta de frutas y/o vegetales & 11 & 5.5 \\
\hline Ingesta de menor calorías de las necesarias & 11 & 5.5 \\
\hline Aumento de consumo de alcohol & 4 & 2.0 \\
\hline
\end{tabular}




\section{Descripción del uso de herramientas de eSalud}

De los 320 participantes del estudio, 134 (41.9\%), 81 (25.3\%) y 53 (16.6\%) plantearon que habían usado aplicaciones móviles o medios digitales vinculados con ejercicio físico, regulación emocional y alimentación, respectivamente. En el Gráfico 2, se puede observar cómo varió el uso de estas herramientas de eSalud, durante el confinamiento, en cada uno de los ámbitos anteriormente mencionados. La mayoría señaló que el uso de aplicaciones móviles o medios digitales se había mantenido prácticamente igual antes y durante el confinamiento, tanto para alimentación (67.5\%), como para ejercicio físico (44.4\%) y emociones (71.9\%).

Gráfico 2. Apreciación de cambios en la frecuencia de uso de herramientas de eSalud, vinculadas con hábitos de vida y emociones, durante las primeras semanas de confinamiento por COVID-19.

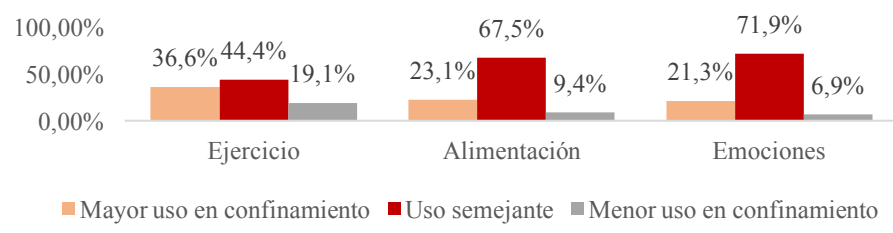

Las herramientas de eSalud utilizadas por los participantes fueron recopiladas mediante preguntas abiertas, en las categorías de alimentación, ejercicio físico y bienestar emocional (dada la extensión del listado no se muestran los datos específicos). YouTube fue la herramienta más utilizada para realizar ejercicio físico (56.3\%), alimentación (26.5\%) y bienestar emocional (17.5\%). La segunda herramienta en uso fue Instagram, apareciendo como respuesta en el $18.4 \%$ de los encuestados para alimentación, 20,2\% para ejercicio físico y $16.3 \%$, para bienestar emocional. En este último aspecto, también es de resaltar el uso de las aplicaciones de Headspace $(12.5 \%)$ y Calm (11.3\%).

\section{Asociación entre el uso de herramientas de eSalud y la valoración subjetiva de los cambios en alimentación, ejercicio físico y emociones durante el periodo inicial del confinamiento por COVID-19.}

En la Tabla 3 se muestran los resultados de la tabla cruzada entre los cambios en la frecuencia de uso de herramientas de eSalud y los cambios en cada uno de los otros parámetros de este estudio (alimentación, ejercicio y emociones). Al comparar (a) modificaciones en el uso de eHealth con (b) los cambios en la alimentación, mediante la prueba $c^{2}$, se obtuvo que había una asociación estadísticamente significativa entre ambas: $c^{2}(4, n=$ $320)=11.75, p=.019$. Con la prueba de comparación de proporciones, se identificó que, dentro del grupo de personas que había disminuido el uso de las herramientas de eSalud vinculadas con alimentación, la proporción de aquellos que habían empeorado su alimentación era significativamente mayor que la de quienes la habían mantenido igual $(p<.05)$. De igual forma, dentro del grupo que mantuvo un uso semejante de herramientas de eSalud relacionadas con alimentación, la proporción de personas que planteó que su alimentación se había mantenido igual fue significativamente mayor que la de quienes la habían empeorado $(p<.05)$. No se observaron otras diferencias significativas.

Al cruzar las variables (a) cambios en la frecuencia de uso de herramientas de eSalud vinculadas a ejercicio físico y (b) cambios en la intensidad/frecuencia de ejercicio físico durante el confinamiento, mediante la prueba $c^{2}$, se obtuvo que había una asociación estadísticamente significativa entre ambas: $c^{2}(4, n=320)=85.35, p=$ .000. Al hacer una prueba de comparación de proporciones, se identificó que, dentro del grupo de personas que había aumentado el uso de las herramientas de eSalud vinculadas al ejercicio físico, la proporción de aquellos que habían incrementado la intensidad/frecuencia de su ejercicio físico era significativamente mayor que la de aquellos que habían disminuido o mantenido el uso de eSalud en este ámbito $(p<.05)$. De igual forma, dentro del grupo que había mantenido un uso semejante de eSalud vinculada al ejercicio físico antes y durante el confinamiento, se observó que la proporción de personas que había mantenido la misma frecuencia/intensidad de 
ejercicio era significativamente mayor que la de quienes lo habían aumentado o disminuido $(p<.05)$. Por último, dentro del grupo de personas que había disminuido el uso de eSalud vinculada al ejercicio físico, se observó que la proporción de individuos que había mermado la frecuencia/intensidad de ejercicio físico era significativamente mayor que la de quienes habían aumentado o mantenido su actividad física $(p<.05)$. El resto de las proporciones no obtuvo una diferencia estadísticamente significativa.

Por su parte, al cruzar las variables (a) cambios en la frecuencia de uso de herramientas de eSalud vinculadas a regulación emocional y (b) cambios en las emociones durante el confinamiento, mediante la prueba $c^{2}$, se obtuvo que no había una asociación estadísticamente significativa entre ambas: $c^{2}(4, n=320)=5.41, p=.25$. Tampoco se observaron diferencias al hacer la prueba de comparación de proporciones (Tabla 3).

Tabla 3. Asociación entre cambios en estilo de vida y emociones y cambios en uso de eSalud durante el confinamiento por COVID-19.

\begin{tabular}{lccc}
\hline Cambios uso de eSalud & \multicolumn{4}{c}{ Cambios estilo de vida y emociones } \\
\hline Alimentación & Mejor $\left(A_{1}\right)$ & Igual $\left(B_{1}\right)$ & Peor $\left(C_{1}\right)$ \\
Mayor & 28 & 24 & 22 \\
Igual & 75 & $91\left(\mathrm{C}_{1}\right)$ & 50 \\
Menor & 9 & 6 & $15\left(B_{1}\right)$ \\
Ejercicio físico & Mayor $\left(A_{2}\right)$ & Igual $\left(B_{2}\right)$ & Menor $\left(C_{2}\right)$ \\
Mayor & $53\left(B_{2} C_{2}\right)$ & 24 & 40 \\
Igual & 23 & $49\left(A_{2} C_{2}\right)$ & 70 \\
Menor & 2 & 1 & $58\left(A_{2} B_{2}\right)$ \\
Estado emocional & Mejor $\left(A_{3}\right)$ & Igual $\left(B_{3}\right)$ & Peor $\left(C_{3}\right)$ \\
Mayor & 12 & 27 & 29 \\
Igual & 29 & 127 & 74 \\
Menor & 4 & 11 & 7 \\
\hline
\end{tabular}

Nota: para cada par significativo ( $p \leq .05$ ), la clave (letra) de la categoría con la columna con la menor proporción aparece en la columna de la categoría con la mayor proporción.

\section{Resultados de las retroalimentaciones sobre "Guía de Buenos Hábitos: Cuidando mi Salud durante el Confinamiento"}

Solo 31 participantes completaron el cuestionario de Retroalimentaciones sobre "Guía de Buenos Hábitos: Cuidando mi Salud durante el Confinamiento". Las puntuaciones promedio en torno a la calidad global de cada una de las secciones de esta guía, en base a un puntaje mínimo de 1 y un máximo de 5, fueron: 4.77 para las sugerencias alimentarias; 4.71 para las dirigidas a mejorar el bienestar emocional; 4.52 para las de ejercicio. Al cuestionar sobre qué tanto habían implementado las sugerencias dadas en cada sección, se obtuvo lo presentado en la Tabla 4. En general, la mayoría de los que ofrecieron su retroalimentación, aplicaron las retroalimentaciones de las secciones. La sección más implementada fue la de bienestar emocional (aplicadas por 80.7\%). 
Tabla 4. Frecuencia de uso de las sugerencias alimentarias, de ejercicio físico y de bienestar emocional ofrecidas en Guía de Buenos Hábitos: Cuidando mi Salud durante el Confinamiento.

\begin{tabular}{|c|c|c|c|c|c|}
\hline & \multicolumn{5}{|c|}{ Frecuencia de uso de las sugerencias guía } \\
\hline & $\mathbf{0}$ & $1-2 d$ & 3-4d & 4-5d & $\geq 6 \mathrm{~d}$ \\
\hline $\begin{array}{l}\text { Sección de } \\
\text { alimentación (\%) }\end{array}$ & 16.1 & 0.0 & 32.3 & 22.6 & 29.0 \\
\hline $\begin{array}{l}\text { Sección de bienestar } \\
\text { emocional (\%) }\end{array}$ & 19.4 & 6.5 & 12.9 & 25.8 & 35.5 \\
\hline $\begin{array}{l}\text { Sección de ejercicio } \\
\text { físico }(\%)\end{array}$ & 25.8 & 3.2 & 12,9 & 19.4 & 38.7 \\
\hline
\end{tabular}

Nota: $\mathrm{d}=$ días.

\section{DISCUSIÓN}

La pandemia de la COVID-19 ha supuesto cambios en los hábitos de vida de muchas personas, tanto a nivel de alimentación, como de actividad física y salud mental, consecuencia del estricto confinamiento llevado a cabo por muchos países (Ramírez et al., 2021). El confinamiento ha contribuido a un mayor aislamiento social que perjudica al bienestar mental de la población (Wilke et al., 2020). En el presente trabajo se analiza este impacto en una población hispanohablante, mayoritariamente procedente de República Dominicana, presentando estos datos gran interés, ya que la mayoría de los disponibles en bibliografía se corresponden con población europea (Bennet et al., 2021).

En el ámbito de la alimentación, los resultados muestran que la pandemia ha supuesto un interés mayor por la alimentación y la necesidad de mejorar los hábitos alimentarios. Esto es importante, ya que gran parte de los pacientes que han requerido de hospitalización en las unidades de cuidados intensivos han sido pacientes con sobrepeso y obesidad, siendo la obesidad uno de los factores de riesgo más importantes para el agravamiento de la infección por el virus SARS-CoV-2 (Bellido et al., 2020).

Un dato interesante que resultó en esta investigación fue el aumento de la comida casera y la elección de alimentos más saludables (Tabla 2) durante el confinamiento. En esta misma línea de estudio, Błaszczyk-B benek et al. (2020) observaron que los polacos habían reducido significativamente el consumo de comida fuera de casa. Además, Rodríguez-Pérez et al. (2020) hallaron que los españoles habían aumentado su adherencia a la dieta mediterránea durante el confinamiento.

No obstante, en el presente estudio y en otros (Błaszczyk-B benek et al., 2020) se señalan, como efectos negativos del confinamiento, un aumento de ingestas por día y el consumo de alimentos con menor calidad nutricional. Esto puede deberse, en parte, a las restricciones de movimiento y a la escasez de algunos productos frescos en algunos picos de la pandemia (Bennet et al., 2021; Carducci et al., 2021).

Respecto a la actividad física, se ha observado una disminución, debido, principalmente, a que las políticas de cierre de gimnasios, parques y espacios al aire libre ha influido negativamente sobre la actividad física y la autoestima de la población (López-Bueno et al., 2020; Sang et al., 2021). Esta reducción de la actividad física ha Ilevado a reorganizar la forma de hacer ejercicio físico en el hogar (Chen et al., 2021). Dichos autores proponen que el ejercicio en casa, utilizando ejercicios seguros, sencillos y fáciles de implementar es muy adecuado para mantener la aptitud y nivel de actividad física. No obstante, al igual que en el caso de la alimentación, la disminución de la actividad física parece menor en personas que se apoyaron en el uso de herramientas de eSalud, siendo de nuevo YouTube e Instagram, las plataformas preferidas. Esto es coherente con lo encontrado por Borrega-Mouquinho et al. (2021), quienes comprobaron una mejoría en la actividad física e, incluso, en la ansiedad, depresión, el estrés y la resiliencia, en quienes participaron en su programa de ejercicio, de moderada y alta intensidad, transmitido por YouTube durante el confinamiento. Sin embargo, como se comenta en el estudio de Kanstrup et al. (2020), si el uso de herramientas de eSalud no viene apoyado por un facilitador o profesional de 
la salud que genere interacción y apoyo social, las personas, por sí mismas, no tienden a comprometerse adecuadamente a acceder a estas plataformas.

En cuanto al área de la salud emocional, se evidenciaron menor percepción de cambios del estado anímico, así como en la necesidad de apoyarse en herramientas de eSalud. Resulta llamativo cuando el uso de las herramientas de teleconferencia ha crecido exponencialmente desde el confinamiento, pero quizás su empleo se vio afectado por la falta de percepción del apoyo que dieron estas herramientas durante este periodo (RodríguezGonzález et al., 2020). Por otro lado, es verdad que el uso de herramientas de eSalud para salud mental es menos conocida por la población general, y que sería necesario fomentar su uso aún en población sana, para evitar posibles efectos que deterioren la salud mental a largo plazo (Briffault, et al., 2018). Por tanto, es necesario fomentar el uso de aplicaciones como Headspace o Calm, utilizadas en algunos estudios científicos, y cuyos criterios de calidad son mayores al de otras herramientas (Clarke \& Draper, 2019; Salehzadeh Niksirat et al., 2017). Estamos de acuerdo con que estas herramientas vienen a completar el papel del terapeuta y que pueden ser de gran ayuda para el fomento de hábitos de bienestar mental y de estilo de vida.

\section{CONCLUSIONES}

La mayoría de los participantes planteó que su alimentación y estado emocional se habían mantenido semejantes antes y durante el confinamiento por COVID-19, mientras que su ejercicio físico había disminuido en intensidad/frecuencia. El uso de eSalud podría ser un aliado para mejorar los hábitos alimentarios y el ejercicio. En el ámbito de la salud mental, resulta necesario seguir profundizando con investigaciones longitudinales sobre el tema y con estudios mixtos que investiguen si estas herramientas pueden apoyar el papel del terapeuta, especialmente en épocas de aislamiento social.

\section{REFERENCIAS BIBLIOGRÁFICAS}

Bellido, D., Tejera, C., Bao, A. S., y Porca, C. (2020). Obesidad y COVID-19. Revista Española de Nutrición Humana y Dietética, 24(Sup 1), 20-21.

Bennett, G., Young, E., Butler, I., y Coe, S. (2021). The Impact of Lockdown During the COVID-19 Outbreak on Dietary Habits in Various Population Groups: A Scoping Review. Frontiers in Nutrition, 8, 53. https://doi.org/10.3389/fnut.2021.626432

Błaszczyk-B benek, E., Jagielski, P., Bolesławska, I., Jagielska, A., Nitsch-Osuch, A., y Kawalec, P. (2020). Nutrition behaviors in Polish adults before and during COVID-19 lockdown. Nutrients, 12(10), 3084. https://doi.org/10.3390/nu12103084

Borrega-Mouquinho, Y., Sánchez-Gómez, J., Fuentes-García, J. P., Collado-Mateo, D., y Villafaina, S. (2021). Effects of High-Intensity Interval Training and Moderate-Intensity Training on Stress, Depression, Anxiety, and Resilience in Healthy Adults During Coronavirus Disease 2019 Confinement: A Randomized Controlled Trial. Frontiers in Psychology, 12, 270. 10.3389/fpsyg.2021.643069

Briffault, X., Morgiève, M., y Courtet, P. (2018). From e-Health to i-Health: Prospective reflexions on the use of intelligent systems in mental health care. Brain sciences, 8(6), 98. https://doi.org/10.3390/brainsci8060098

Carducci, B., Keats, E. C., Ruel, M., Haddad, L., Osendarp, S. J. M., y Bhutta, Z. A. (2021). Food systems, diets and nutrition in the wake of COVID-19. Nature Food, 2(2), 68-70. https://doi.org/10.1038/s43016-02100233-9

Chen, P., Mao, L., Nassis, G. P., Harmer, P., Ainsworth, B. E., y Li, F. (2020). Coronavirus disease (COVID-19): The need to maintain regular physical activity while taking precautions. Journal of Sport and Health Science, 9, 103-104. https://doi.org/10.1016/j.jshs.2020.02.001

Clarke, J., y Draper, S. (2019). Intermittent mindfulness practice can be beneficial, and daily practice can be harmful. An in depth, mixed methods study of the "Calm" app's (mostly positive) effects. Internet Interventions, 19, 100293. https://doi.org/10.1016/j.invent.2019.100293 
Constandt, B., Thibaut, E., De Bosscher, V., Scheerder, J., Ricour, M., y Willem, A. (2020). Exercising in times of lockdown: an analysis of the impact of COVID-19 on levels and patterns of exercise among adults in Belgium. International journal of environmental research and public health, 17(11), 41-44. https://doi.org/10.3390/ijerph17114144

Hernández Sampieri, R., Fernández Collado, C., y Baptista Lucio, M. D. P. (2014). Metodología de la Investigación (6ta ed.). México, D.F.: McGraw-Hill/interamericana Editores, S.A. de C.V.

Jeong, H., Yim, H. W., Song, Y. J., Ki, M., Min, J. A., Cho, J., y Chae, J. H. (2016). Mental health status of people isolated due to Middle East respiratory syndrome. Epidemiology and Health, 38, e2016048. https://doi.org/10.4178/epih.e2016048

Kanstrup, A. M., Bertelsen, P. S., y Knudsen, C. (2020). Changing Health Behavior with Social Technology? A Pilot Test of a Mobile App Designed for Social Support of Physical Activity. International journal of Environmental research and Public health, 17(22), 8383. https://doi.org/10.3390/ijerph17228383

López-Bueno, R., Calatayud, J., Andersen, L. L., Balsalobre-Fernández, C., Casaña, J., Casajús, J. A., Smith, L., y López-Sánchez, G.F. (2020). Immediate impact of the COVID-19 confinement on physical activity levels in Spanish adults. Sustainability, 12(14), 5708; https://doi.org/10.3390/su12145708

Marchant, G., Bonaiuto, F., Bonaiuto, M., y Guillet-Descas, E. (2021). Exercise and physical activity eHealth in COVID-19 pandemic: a cross-sectional study of effects on motivations, behavior change mechanisms, and behavior. Frontiers in Psychology, 12, 618362. https://doi.org/10.3389/fpsyg.2021.618362

Organización Mundial de la Salud. (2020). Actualización de la estrategia frente a la COVID-19. Ginebra: OMS.

Ramírez, F. B., Misol, R. C., Alonso, M. D. C. F., y Tizón, J. L. (2021). Pandemia de la COVID-19 y salud mental: reflexiones iniciales desde la atención primaria de salud española. Atención primaria, 53(1), 89-101. https://doi.org/10.1016/j.aprim.2020.06.006

Rodríguez-González, R., Facal, D., Martínez-Santos, A. E., y Gandoy-Crego, M. (2020). Psychological, social and health-related challenges in Spanish older adults during the lockdown of the COVID-19 first wave. Frontiers in Psychiatry, 11, 1393. 10.3389/fpsyt.2020.588949

Rodríguez-Pérez, C., Molina-Montes, E., Verardo, V., Artacho, R., García-Villanova, B., Guerra-Hernández, E. J., y Ruíz-López, M. D. (2020). Changes in dietary behaviours during the COVID-19 outbreak Confinement in the Spanish COVIDiet Study. Nutrients, 12, 1730. https://doi.org/10.3390/nu12061730

Salehzadeh-Niksirat, K., Silpasuwanchai, C., Ahmed, M., Cheng, P., y Ren, X. (2017). A framework for interactive mindfulness meditation using attention-regulation process. In Proceedings of the $2017 \mathrm{CHI}$ Conference on Human Factors in Computing Systems (pp. 2672-2684). https://doi.org/10.1145/3025453.3025914

Sang, X., Menhas, R., Saqib, Z. A., Mahmood, S., Weng, Y., Khurshid, S., Iqbal, W., y Shahzad, B. (2021). The psychological impacts of covid-19 home confinement and physical activity: A structural equation model analysis. Frontier Psychology, 11, 614-770. https://doi.org/10.3389/fpsyg.2020.614770

Wilke, J., Mohr, L., Tenforde, A. S., Vogel, O., Hespanhol, L., Vogt, L., Verhagen, E., y Hollander, K. (2020). Activity and health during the SARS-CoV2 pandemic (ASAP): study protocol for a multi-national network trial. Frontier Medicine, 7, 302. https://doi.org/10.3389/fmed.2020.00302

Yang, X., Song, B., Wu, A., Mo, P. K., Di, J., Wang, Q., Lau, J. T. F, y Wang, L. (2021). Social, cognitive, and eHealth mechanisms of COVID-19-related lockdown and mandatory quarantine that potentially affect the mental health of pregnant women in china: cross-sectional survey study. Journal of Medical Internet Research, 23(1), e24495. https://doi.org/10.2196/24495 
